\title{
Quantification of Thermal Aging in Cable Insulation Using Contact Resonances of a U-shaped Atomic Force Microscope Probe
}

\author{
Ehsan Rezaei ${ }^{1}$, Pradeep Ramuhalli ${ }^{2}$, Samuel W. Glass ${ }^{2}$, Leonard S. Fifield ${ }^{2}$ and Joseph A. Turner ${ }^{1}$ \\ 1. Mech. and Materials Eng., Univ. of Nebraska-Lincoln, W342 NH, Lincoln, Nebraska 68588, USA \\ 2. Pacific Northwest National Laboratory, Richland, Washington 99352, USA
}

Nuclear power plants (NPPs) currently comprise about $20 \%$ of the U.S. energy portfolio. Many of these NPPs are currently licensed to operate up to 60 years past their original design and exhibit high capacity factors and low capital investment requirements. As a result, there is currently great interest in extending the operating life of current NPPs to 80 years through a second round of license renewals. However, safety is of utmost importance for such extensions and passive components, including electrical cables, are a key element of the safe operation of NPPs. Life extension is thus intimately connected with electrical cable safety so that an NPP can function as designed through end-of-licensed life. Cables in NPPs can traverse multiple environments, with variations in temperature, humidity, and radiation dose. A clear understanding of cable degradation mechanisms is very important for the assessment of cable remaining life. In the last several years, major progress has been made to enhance the understanding of thermal and radiation damage in cable insulation [1]. The damage to the polymer insulation first causes it to lose its dissipative behavior (through viscoelastic changes) which can result in the growth of microcracks. As the structural integrity of the cable is reduced, the damage can eventually reach the cable core and may compromise the integrity of the associated electrical network. Condition monitoring approaches can assess bulk insulation behaviour but a full understanding of these tests requires a better understanding of cable aging mechanisms under the influence of environmental stressors (moisture, contaminants, oxidation). Thus, it is critical to quantify the damage mechanisms at the length scale of the polymer network; consequently nano-to-micro-to-macroscale measurement techniques are needed.

Contact resonance atomic force microscope (CR-AFM) methods are relatively new measurement techniques used to quantify the elastic and viscoelastic properties of numerous materials [2,3]. More recently, AFM thermalevers have been developed to allow local heating of samples and the resonances of these probes are much more complex. These probes have one distinct advantage over rectangular AFM probes in that specific modes allow in-plane and out-of-plane tip-sample motion to be excited independently at the same location using a Lorenz force excitation. Here, a commercial thermalever, a $\mathrm{U}$-shaped probe, is used to determine the in-plane and out-of-plane viscoelastic properties at the same location. The CR-AFM approach involves measurement of the resonant frequencies of the AFM probe both for the free case and the case for which the tip is in contact with the sample. A simplified analytical model of the U-shaped probe vibrations [4] is then used to determine the sample properties from the frequency behaviour.

A cable of ethylene propylene rubber (EPR) was aged in an air environment at $140{ }^{\circ} \mathrm{C}$ for 1200 hours. A sample was then removed from the cable and used to perform the CR-AFM measurements. The frequency spectrum when the tip is in contact with EPR is obtained and includes modes for both symmetric (bulk) and asymmetric (shear) flexural vibrations. The resonances are then fit as shown in Fig. 1 using the vibration model [4] in order to obtain the frequency and Q-factor of each peak - these are related to the stiffness and damping of the sample, respectively. Knowledge of free probe frequency spectrum along with the contact spectrum allows the dimensionless contact stiffness and damping to be 
calculated [5], from which the loss tangent for the bulk and shear modes can be determined. Figure 2(A) shows the results of CR-AFM with respect to the distance from the heated surface. The loss tangent has a clear drop near the damaged surface showing that this region has less dissipative material behavior. The results also suggest two length scales of damage that are characterized by the deformation modes. The bulk mode shows a transition before $400 \mu \mathrm{m}$ while the shear mode shows a similar transition before $700 \mu \mathrm{m}$. Figure 2(B) compares Fourier transform infrared spectroscopy (FTIR) at two positions (damaged and undamaged) on the sample. The FTIR peaks illustrate a decrease in the carbonyl group and an increase in hydroxyl groups which has been observed by others as well [1]. It is anticipated that the ability of CR-AFM to quantify spatial variations in viscoelastic loss tangent at such small scales will allow polymer damage degradation to be more clearly understood.

\section{References:}

[1] U.S. Department of Energy Office of Nuclear Energy, Light Water Reactor Sustainability Program, Report M2LW-15OR0404024, September 2015.

[2] D G Yablon, J Grabowski and I Chakraborty, Measurement Science and Technology 25 (2014), p. 055402.

[3] D C Hurley, S E Campbell, J P Killgore, L M Cox and Y Ding, Macromolecules 46 (2013), p. 9396.

[4] E Rezaei and J A Turner, Journal of Applied Physics 119 (2016), article 034303.

[5] S E Campbell, V L Ferguson and D C Hurley, Acta Biomaterialia 8 (2012), p. 4389.
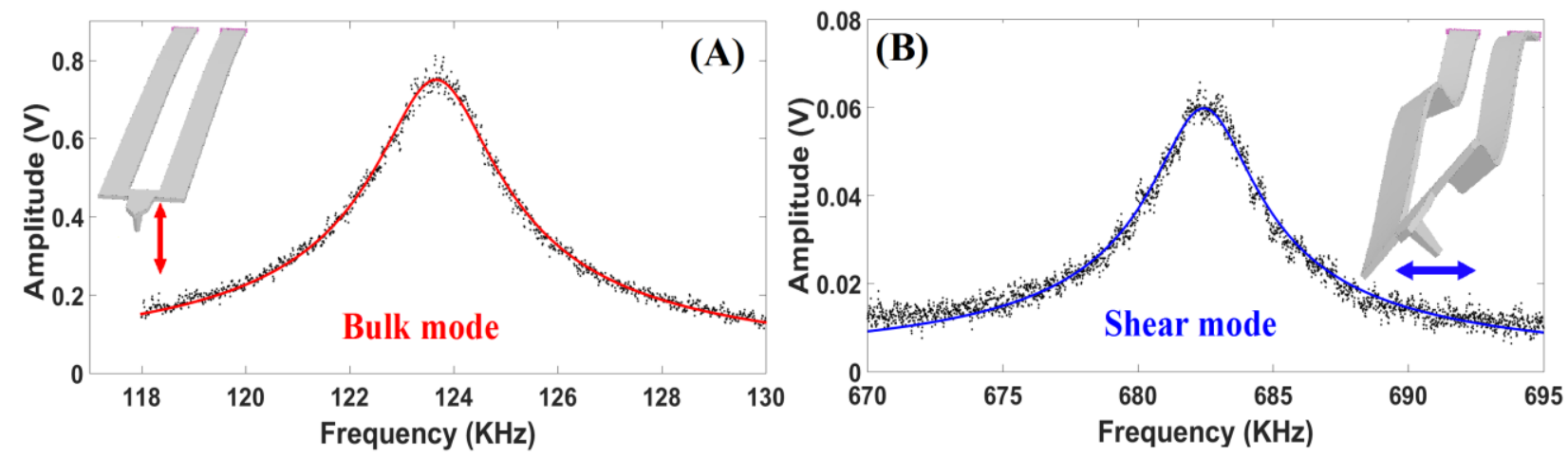

Figure 1. Times CR-AFM peaks measured on EPR: symmetric (A) and asymmetric (B) peaks define the loss tangent for different deformation modes [4].
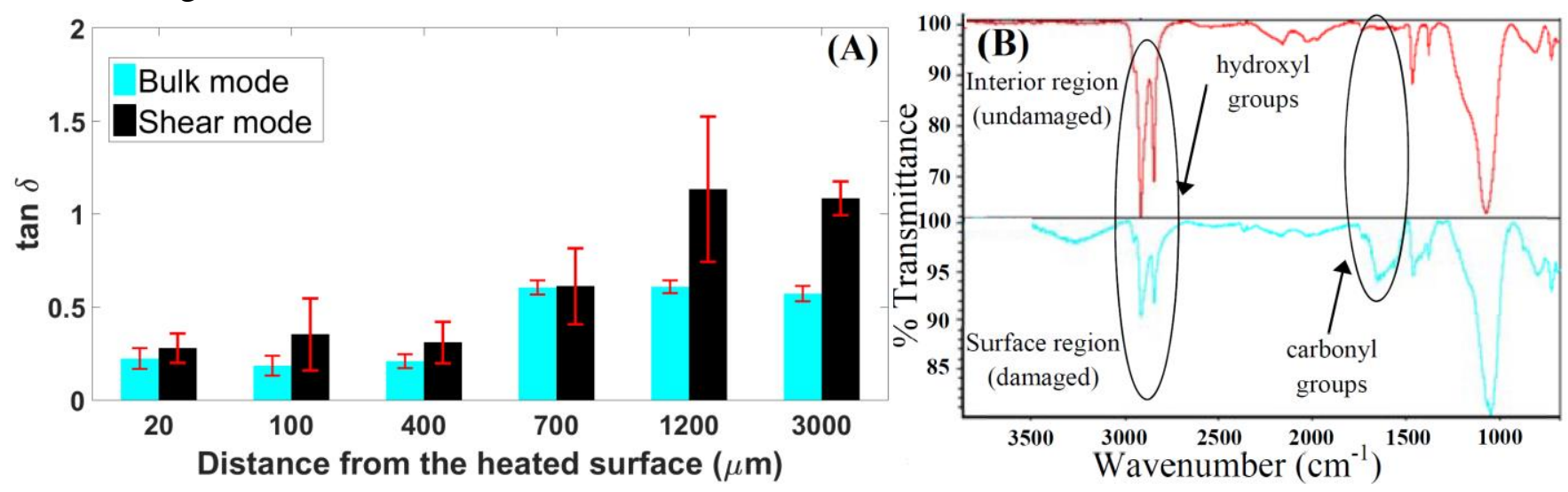

Figure 2. The CR-AFM results (A) show loss tangent for two different types of material deformation behavior (bulk and shear). The FTIR results (B) from an interior position (undamaged) and a surface region (damaged) show clear difference in polymer structure. 